\title{
Comunidades profesionales de aprendizaje: oportunidades de desarrollo profesional en la exclusión y diversidad rural
}

\author{
Alberto Galaz* \\ Universidad Austral de Chile \\ Claudia Contreras** \\ Universidad Austral de Chile \\ Paola Acosta*** \\ Universidad Libre
}

Resumo Las recientes iniciativas oficiales dirigidas al profesor buscan impregnar de nuevos principios, estrategias e instrumentos su desempeño. Pero se trata de significados que buscan desplegarse sobre contextos diversos, colmados de tradiciones que no necesariamente pueden ser coincidentes o abiertamente permeables y que de no ser considerados podrían generar resistencias que obstaculicen el cambio deseado y posterguen los anhelos de contar con reales oportunidades de aprendizaje. En particular, el desarrollo de la escuela y del profesor rural requiere estrategias ambiciosas, que tomando distancia de coordenadas de oposición urbano-rural y de un enfoque del déficit consideren la complejidad de las dinámicas territoriales, multiculturales y lingüísticas. Sobre la base de un análisis crítico-descriptivo este artículo aborda los elementos configurativos del desempeño del profesor rural y evalúa los alcances teórico-prácticos de una estrategia de Comunidades Profesionales de Aprendizaje.

PALAVRAS-CHAVE: Profesor; Escuela rural; Política; Desarrollo profesional; Identidad profesional. 


\title{
Professional Communities for Learning: opportunities of professional development in the exclusion and rural diversity
}

\begin{abstract}
In Chile, the policy for professional development seeks to confer a new basis, strategies and tools to the performance of schools and the teachers' labor. It is related to new concepts and meanings to be applied in different contexts, full of tradition, which not necessarily are the same or open, and if not consider could generate some obstacles for the desire change and which may also interfere with the goal of having real development opportunities. In particular, the development of rural schools and teachers requires ambitious strategies considering the urban-rural opposition and responding appropriately to the difficult dynamics of territory, multiculturalism and linguistics. Through a critical-descriptive analysis this paper reviews the elements shaping the development of a rural school teacher and evaluates the consequences of the application of Professional Communities for Learning from the policy for professional teachers' development.
\end{abstract}

KEYWORDS: Rural school, Policy, Professional development, Professional identity.

\section{Introducción}

Concebidas tradicionalmente desde coordenadas de oposición a lo urbano, las políticas educativas implementadas por el Estado para el mundo rural, salvo excepciones, han transitado desde la no visualización a la asistencialidad. Pero lo rural debiera ser concebido más bien como una dinámica de transformación territorial, cultural y económica permanente, un ethos particular donde el desempeño, la identidad profesional y las necesidades de formación del profesor adquieren connotaciones complejas y singulares (Ferrada, 2015; Peirano, 2015; Galaz, 2011a y Williamson, 2004).

En lo particular, el mejoramiento de la escuela rural y el desarrollo profesional del profesor demandan estrategias ambiciosas que partiendo del reconocimiento de esta complejidad sean suficientemente flexibles para promover aprendizaje organizacional. Al respecto, existe un consenso de que las estrategias tradicionales de formación (capacitación-perfeccionamiento) y evaluación (individual-externa) no se han mostrado efectivas para responder a los desafíos (Montecinos, 2003; OCDE, 2009 y OCDE, 2013).

En este contexto, la reciente promulgación de la Ley de Desarrollo Profesional (LDP) apunta a configurar un nuevo escenario para el ejercicio y la formación inicial y continua de los profesores bajo la adopción de principios que apuntan a concebir que el devenir profesional transcurre en el contexto de una cultura de trabajo colaborativa, situado y bajo la gestión de las propias escuelas. Además, establece nuevas instancias e instrumentos que refuerzan la idea de un desarrollo profesional concebido como un continuum en la trayectoria del profesor. Otros ejes relevantes de la ley son: 
A. Plan de mejoramiento y formación continua de profesores elaborado por la escuela

B. Centro de Perfeccionamiento e Investigaciones Pedagógicas de carácter regional

C. Oferta de formación continua sin fines de lucro

De esta forma se puede evidenciar un doble objetivo en la ley: menos transmisión frontal del conocimiento y aprendizaje individual y más aprendizaje colaborativo e integración de la teoría y la práctica. Lo interesante es que la ley también señala explícitamente que las Comunidades Profesionales de Aprendizaje (CPA) constituyen la estrategia clave a emplear para lograrlo.

Sin embargo, muy pocos estudios han tendido a separar características cuando se analizan posibles factores facilitantes de desarrollo y despliegue de una CPA, lo que puede contraer una gran confusión acerca de lo que debe ser objeto de examen y análisis. La diversidad de enfoques existentes y la tentación de dar recomendaciones también riesgo de que puedan ser asumidas como recetas respecto de ciertos estándares exigibles y no como un proceso de aprendizaje comunitario en el cual confluyen lógicas y dinámicas divergentes.

De esta forma, en consideración de las múltiples condiciones bajo las cuales se despliega el ejercicio docente rural en Chile, como así también, de la implementación de una política que busca re-configurar bajo nuevos significados el escenario actual de formación promoviendo abiertamente el desarrollo de CPA, se ha configurado un escenario de alta complejidad donde la ausencia de comprensión profunda de los procesos podría obstaculizar o inclusive generar resistencias al cambio deseado. sino se asume un enfoque también complejo que permita comprender y visualizar las múltiples dimensiones involucradas.

$\mathrm{Al}$ respecto nos preguntamos ¿Cuáles han sido los elementos configurativos predominantes en la definición del desempeño de los profesores rurales? ¿Qué debemos entender por desarrollo profesional efectivo? ¿Qué evidencias o antecedentes podrían sostener la promoción de CPA en la escuela rural? ¿Hacia qué finalidades debieran orientar los profesores rurales el desarrollo de una CPA?

Sobre la base de un análisis crítico-descriptivo este artículo se dirige a la identificación de los elementos configurativos del desempeño del profesor rural y a la evaluación de los alcances teórico-prácticos de una estrategia de Comunidades Profesionales de Aprendizaje.

A nuestro juicio la relevancia del análisis que se propone radica en que:

A. Se contextualiza en un escenario inédito de avance en el desarrollo profesional del profesor y de condiciones propicias para el despliegue de estrategias colaborativas.

B. Permite evidenciar las coordenadas singulares que asume el desempeño del profesor rural. 
C. Busca contribuir como correlato o contrastación a la articulación de las propuestas teóricas de desarrollo de CPA y a los instrumentos e instancias de desarrollo profesional que se promueven con las nuevas normativas.

\section{La escuela rural: enfoques de análisis}

Según datos del Ministerio de Educación de Chile (Mineduc) las escuelas rurales en el país alcanzan un número de 3.654, lo que representa el 31\% del total nacional (Mineduc, 2016). No resulta difícil comprender que la mayor presencia de éstas se establezca hacia las zonas sur y norte, tomando como referencia la zona central y particularmente la Región Metropolitana, aunque en números concretos es la zona sur y particularmente las regiones VIII, IX, X y XIV las que poseen mayor representación.

Sobre estas cifras es clave mencionar que el número total de estudiantes matriculados en escuelas rurales al año 2016 alcanza la cifra de 270.956 (aproximadamente un 7,6\% de la matricula nacional) y que alrededor de 26.285 profesores se desempeñan en ellas (11,7\% del total nacional). Cuando recurrimos al criterio de identificación por dependencia advertimos que la escuela rural, los alumnos y el cuerpo docente se concentran fundamentalmente en el sector público municipal (76\%, 71\% y $78 \%$ respectivamente). Cabe señalar además que en su mayor parte estas escuelas son multigrado y que el $54 \%$ posee un número de 4 o más profesores y el $46 \%$ restante entre 1 y 3 . Otro dato interesante es que un $63 \%$ de las escuelas posee una matrícula menor a 50 estudiantes.

Pero más allá de estas estadísticas cabe preguntarse por aquellos aspectos distintivos de una escuela rural. Según Williamson (2004) la primera tentación es recurrir a la clásica oposición de lo urbano y lo rural. En segundo lugar a la pobreza, como factor inherente. Sobre este último aspecto, el Programa de las Naciones Unidades para el Desarrollo (PNUD) en su informe de Desarrollo Humano rural (2008) señala:

Somos depositarios de una imagen que vincula dichas formas de vida al atraso y al carácter autónomo de sus lógicas de reproducción social: estructura familiar extensa, precarias condiciones de vida, equipamiento deficitario de las viviendas, formas tradicionales de consumo cultural y estrategias clásicas para generar los medios de subsistencia [...] (PNUD, 2008, p. 70).

La pobreza aún permanece como factor definitorio de las zonas rurales. Aproximadamente el 22\% de las personas que habitan en zonas rurales de nuestro país se encuentran en esta condición y el $15 \%$ en pobreza extrema, cifras que representan más del doble que las encontradas en zonas urbanas (CASEN, 2015). Pero el mapa rural identifica coordenadas de orientación más dinámicas y complejas de lo esperado, toda vez que en muchas áreas también es posible apreciar avances, por ejemplo, en cobertura escolar, infraestructura, conectividad, etc. en tanto también se experimentan nuevas formas de exclusión.

En los análisis sobre la escuela rural también es posible advertir la prevalencia de un enfoque que la concibe bajo esquemas excluyentes, una visión que tiende 
a homologarlas a otras que comparten estas condiciones. Leyton (2015), fundamenta esta prevalencia en razón de iniciativas asistencialistas, es decir, aquellas que, salvo el Programa de Mejoramiento de la Calidad y Equidad de la Educación para Educación Básica Rural (MECE/Básica/Rural) del año 1992 y, en menor forma, el Programa de Educación Intercultural Bilingüe (PEIB) 1996, no hacen reconocimiento de la ruralidad como espacio y condición específica de comprensión.

En palabras de Peirano,

\begin{abstract}
"Es así que estas comunidades, lamentablemente, se encuentran en una suerte de rincón imperceptible ante aquellos entes encargados en la toma de decisiones, donde hasta ahora, lo rural ha ocupado un espacio acotado y prácticamente invisible en la política educativa de Chile" (2015:55).
\end{abstract}

La educación rural no posee reconocimiento como modalidad o nivel específico del sistema y si bien los programas ejecutados desde el 1992 y decreto No968 (2012) reconocen sus singularidades no han sido suficientes para asegurar o sostener su desarrollo. Con todo, entre el año 2007 y 2014 se han cerrado aproximadamente 600 escuelas rurales y cabe preguntarse cuánto de este fenómeno es explicado por la no visualización de lo rural como espacio específico de desarrollo. Las explicaciones tradicionales corren a favor de la migración campo-ciudad y al mayor número de expectativas de desarrollo en áreas urbanas. Cabe preguntarse entonces, ¿por qué el Estado debiera intentar frenar una situación que a todas luces parece inevitable?

Las respuestas nos remiten al sentido de la política, a los enfoques de su diseño, a su naturaleza técnico-instrumental o social. El no diseño de políticas señala Guerra (2013) también es un acto de política y si el sujeto rural desaparece esta desaparición no es inocente, representa opciones políticas declaradas, ante las cuales es necesario pronunciarse.

De esta forma, definir lo rural bajo enfoques y categorías exclusivas parece ser limitante en vista de las evidencias que configuran un escenario en cual se entrecruzan diversos componentes lingüísticos, culturales y económicos. Las clásicas definiciones se ven superadas por lo que se ha denominado como compleja dinámica de transformación territorial, ultural y económica donde las contradicciones, subordinaciones e insubordinaciones no son ajenas, un ethos particular, un espacio de límites difusos (Williamson, 2004 y Peirano, 2015).

\title{
El profesor rural, contexto y desafíos de formación
}

Al igual que otros contextos y territorios, el mundo rural, sus comunidades $\mathrm{y}$ actores se han visto impactados por los procesos de globalización y por las transformaciones en el orden productivo y distributivo que contraen. Ante los acuerdos estratégicos de escala mundial por ejemplo, el lugar ocupado ha sido de meros espectadores. Por su parte, los efectos se han dejado sentir en múltiples dimensiones, tales como; el uso del suelo, la producción de bienes exportables, la introducción y uso de nuevas tecnologías, la re-organización espacial del territorio, los estilos de vida y consumo, por nombrar algunos. (Pino, 2004 y PNUD, 2008). 
Sin embargo, estos cambios también han impactado en las labores cotidianas, simplificándolas en algunos aspectos, complejizándolas en otros (PNUD, 2008). En palabras de Thomas y Hernández (2004) se trata de la constatación de las dos caras de un mismo modelo hegemónico de desarrollo, por una parte económico y por otra sociocultural, esta última concebida como un fenómeno de penetración de principios de relacionalidad particularmente del individualismo, la competencia y el consumismo. Pero a la creciente incertidumbre generada se ha opuesto una mayor valorización de lo rural, concebido como expresión de una identidad con base en la cultura local y en los patrimonios de distinta índole.

Cabe preguntarse entonces por los impactos específicos en la forma en que se concibe actualmente el rol del profesor rural. Para Egert (2007) los referentes de construcción aluden a un fuerte sentido de pertenencia a la comunidad, como así también, a un no reconocimiento de su condición profesional. Si bien este último factor es transversal a muchos profesores, independientemente si es urbano o rural, este se ve incrementado en razón de su condición de mayor pobreza, y por sobre todo, la diversidad étnica de sus estudiantes.

Así, la diversidad aparece como un factor clave, no solo porque desafía cotidianamente los saberes profesionales, sino porque cuestiona las bases políticas de su formación. Al respecto, el informe de la OREALC-UNESCO (2013) al entregar antecedentes y criterios para la elaboración de políticas de formación para docentes rurales asume como debilidad concebir su desempeño y preparación bajo criterios no integradores de todas aquellas particularidades y dinámicas de lo rural.

Como evidencia de esta complejidad, Ferrada (2015) propone un cuadro muy ilustrativo de escenarios de desempeño (Tabla No1). En su propuesta se articulan tres dimensiones (Cultura, Territorio y Lengua) en un contexto de alta vulnerabilidad y pobreza. Así es posible obtener 16 distintos contextos. En este mismo sentido, al referir exclusivamente a lo rural como eje articulador estos no parecen ser menos numerosos o complejos.

Tabla 1: Diversidad del desempeño laboral del profesorado

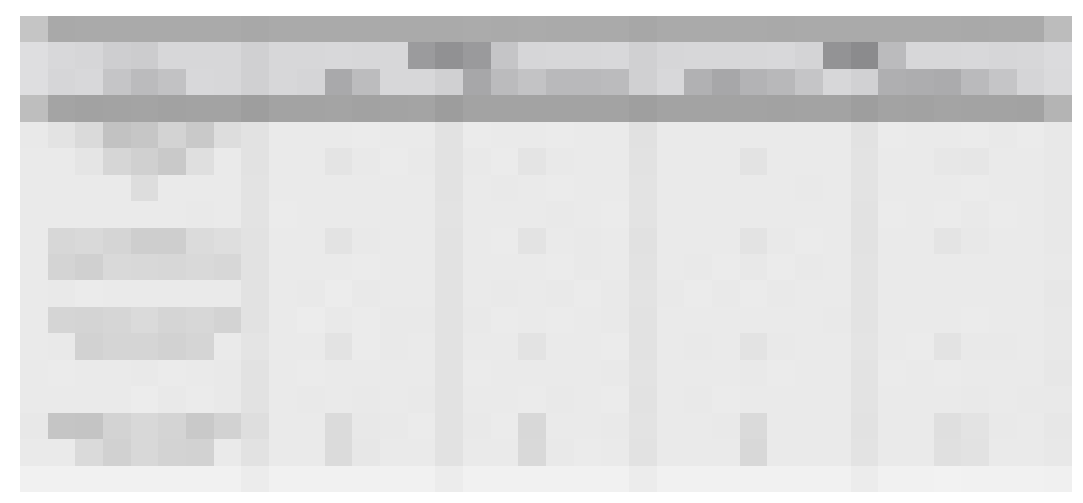

Fuente: Ferrada, 2015 
Cabe preguntarse por la forma en que el sistema educativo ha respondido a estas complejidades y cómo ha generado las condiciones necesarias para que escuelas $\mathrm{y}$ profesores puedan responder de forma pertinente.

La pregunta es capciosa y la respuesta es consabida, dotándose de un discurso progresista la normativas han tendido fundamentalmente en su diseño a prescindir de los actores y a adherir a modelos y enfoques tecnicistas que se han traducido más bien en mayores niveles de estandarización de los desempeños (Marco de la Buena Enseñanza y Estándares Pedagógicos), de control (evaluaciones externas e individuales), prescripción curricular y oportunidades de formación con foco en la capacitación necesaria para la aplicación de los instrumentos oficiales. En palabras de Thomas y Hernández (2004) se trata ante todo de un sistema educativo que imposibilita responder inteligentemente a las distintas realidades.

Las oportunidades de desarrollo profesional para los profesores rurales han tenido en los Microcentros la apuesta central del Mineduc. Su origen radica en el Programa Mece Rural y en una experiencia similar realizada en Colombia a inicio de los años 90'. Se trata de una estrategia particular para las escuelas multigrados, especialmente para las uni, bi y tri docente. En dicha instancia los profesores participantes se reúnen mensualmente con la finalidad de compartir sus experiencias docentes, intercambiar materiales, formular proyectos de mejoramiento, diseñar prácticas curriculares con el apoyo de supervisores del Mineduc (Avalos, 2016).

Los Microcentros rurales recogen las orientaciones teóricas más progresistas sobre el desarrollo y aprendizaje profesional del profesor, al considerar como principio de funcionamiento el trabajo colaborativo, el análisis reflexivo de experiencias, el estudio y diseño de propuestas en base a la identificación de necesidades locales, etc. Pero así también expresan algunas de las principales contradicciones del diseño de política, educativa, particularmente en aquello referido a su despliegue o implementación. Por ejemplo, es curioso que su alcance sea solo para profesores de escuelas multigrado con un máximo de 3 profesores y que no se trate de una instancia que pudiera ser replicada para el total, con ello, actualmente, más del $50 \%$ de los profesores rurales de aproximadamente 1200 escuelas no cuentan con esta oportunidad ${ }^{1}$. Así también, las 2 horas mensuales asignadas para la reunión parecen a todas luces un tiempo insuficiente en razón de las distancias que se deben considerar para llegar al lugar de reunión (generalmente es un profesor de una de las escuelas participantes el que sirve de anfitrión) y esto sin dimensionar la complejidad de las temáticas a abordar. Por último, si consideramos que se trata de reuniones de carácter técnico pedagógicas que cuentan con las orientaciones de un supervisor del Mineduc, es difícil prever que las necesidades más sentidas o emergentes de las escuelas puedan ser abordadas profusamente.

\section{Desarrollo Profesional del profesor: Coordenadas para una estrategia efectiva}

Que los profesores se constituyan en profesionales colaborativos, autónomos y constructores de conocimiento y que esta constitución es un proceso de desarrollo que ocurre a lo largo de la vida, involucrando dimensiones personales, sociales 
y profesionales, puede ser identificado como eje sobre el cual existe el más amplio acuerdo (Day, 2011).

Según Korthagen (2010), este desarrollo profesional debe permitir a los profesores visualizar y sentir que son conscientes del hecho de que deben abandonar zonas de bienestar y que deben asumir crecientes niveles de incertidumbre, tomar riesgos y decisiones. Pero, la efectividad del desarrollo profesional es tema permanente de discusión y no han sido menores los intentos por identificar y clasificar las estrategias que se estiman pertinentes.

En lo específico cada una de ellas puede ser interrogada en relación a los principios epistémicos que la configuran, particularmente en lo relativo a:

¿Cómo conocen los profesores?

¿Cuáles son los conocimientos necesarios para una enseñanza efectiva?

¿Quién genera o produce el conocimiento de la enseñanza?

Una primera aproximación distingue estrategias externas de perfeccionamiento de aquellas que se realizan entre pares, de forma mixta o combinada (OCDE, 2013). Otra distingue entre actividades de capacitación y de colaboración (Ávalos, 2011 y OREALC-UNESCO, 2013). Es consabido que las estrategias de "capacitación y perfeccionamiento" parten del supuesto déficit del profesor y apuntan a su rol pasivo y receptivo frente a las innovaciones. La principal debilidad de estas aproximaciones ha sido la de pecar en artificialidad al pretender definir la enseñanza como una síntesis de conductas medibles. A pesar de sus debilidades su oferta ha sido predominante (OCDE, 2009 y 2013).

Frente a las limitaciones señaladas la búsqueda de experiencias no se ha hecho esperar. Las investigaciones realizadas constatan que el desarrollo profesional concebido como una práctica cooperativa en la cual los maestros comparten recursos y reflexiones se ha demostrado esencial para la persistencia y éxito de las innovaciones (Montecinos, 2003). Así, debemos preguntarnos por el motivo de éxito de estas estrategias. Al respecto, coincidimos con las razones dadas por Houssaye (2005) sencillamente por su simplicidad:

A. No existe alternativa a la primacía del contacto personal, los profesores tienen que renovar sus conocimientos y habilidades, pero también es preciso que dispongan y se les otorguen oportunidades y espacios para reunirse, a título individual o colectivo, para dar o recibir ayuda, o simplemente para conversar sobre el sentido del cambio.

B. La segunda coloca su acento en la descripción del contexto actual, en tanto advierte que, en unos tiempos diversos, complejos y turbulentos, las relaciones, la construcción de comunidades, más allá del propio centro, son vitales para mejorar la calidad del aprendizaje.

C. La tercera razón alude a la posibilidad de generar una reflexión pertinente por parte del profesor. Como señala: "el que se forma, para formarse debe partir de sus propias incertidumbres, de sus propios cuestionamientos" (p. 109). 
En Chile, los análisis realizados sobre la promoción de trabajo colaborativo tales como; Grupos Profesionales de Trabajo, Redes Pedagógicas, Redes de Maestros constatan que han sido experiencias muy valoradas, particularmente por dos razones; en primer lugar, por la pertinencia de los saberes (metodologías de trabajo, nuevos conceptos disciplinares, instrumentos y guías de trabajo) y, en segundo, por el apoyo, contención y reconocimiento social que otorgan en medio de múltiples demandas y estresantes jornadas de trabajo (Montecinos, 2003; Cornejo, 2007 y Galaz, 2011b).

\section{Comunidades Profesionales de Aprendizaje: antecedentes, definición y supuestos}

Después de los cambios educativos realizados en Estados Unidos en la década de los 60' una de las principales conclusiones obtenidas fue que, a fin de lograr una reforma efectiva y sustentable se debía contar con el protagonismo y participación de los profesores. La clave de este giro fue el principio planteado por Senge (2004) relativo a que "las organizaciones aprenden" (Vescio, Ross y Adams, 2008; Krichesky y Murillo, 2011). En el contexto escolar la noción de organizaciones que aprenden será posteriormente fusionada a aquella de comunidad profesional de profesores (derivada de las posturas contraculturales y anti capitalistas en educación) dando origen a su actual denominación de Comunidades Profesionales de Aprendizaje.

Los autores concuerdan que no existe un consenso sobre la definición de una CPA y que básicamente ella puede aludir a diferentes significados en función de la audiencia interesada, por ejemplo; un proyecto o movimiento de reforma, una cultura de trabajo, un enfoque de gestión, una estrategia o dispositivo de formación o todas ellas (Ho, Lee y Teng, 2016). Menos difícil ha sido identificar sus elementos configurativos. Al respecto se alude a un modelo de desarrollo organizativo-profesional que situado en la escuela:

A. Tiene por finalidad el crecimiento y aprendizaje de profesores y alumnos;

B. Promueve el trabajo colaborativo de profesores, quienes de manera crítica, inclusiva y constante intercambian sus prácticas;

C. Gestiona una construcción situada del conocimiento a partir de la búsqueda de nuevas experiencias, investigando, evaluando e incorporando nuevas ideas;

D. Busca apoyar la innovación y otorgar sentidos reales al cambio educativo.

En base a estos antecedentes estimamos que las CPA invitan a reflexionar al menos en tres dimensiones:

- La gestión y apropiación del conocimiento profesional que se construye,

- Una rearticulación de los actores en el sistema educativo y,

- Las innovaciones y condiciones institucionales de trabajo; por ejemplo, a nivel del tiempo, espacios y recursos. 


\section{Supuestos e implicancias identitarias}

Se ha venido insistiendo y evidenciado que las escuelas son un espacio clave para el aprendizaje profesional, tanto de los profesores en ejercicio como de aquellos que se inician en la profesión. Ha sido sobre la base de estas evidencias que se recomienda realizar procesos tempranos y graduales de inserción (Correa et al., 2013; Korthagen, 2010 y Galaz, 2014). De estas evidencias las CPA asumen dos supuestos: Que el conocimiento, en primer lugar, es una construcción situada resultante de las experiencias vividas día a día por los maestros y, que para alcanzar una mejor comprensión de sus alcances, se requiere en segundo lugar, de la reflexión crítica y socializada.

También se debe hacer mención a las implicancias identitarias que sustentan el desarrollo de una CPA. Autores como Day (2011) y Galaz (2011a y 2015) han referido a los impactos que sobre el autoconcepto o concepción de Si adquiere el reconocimiento que otorga el grupo profesional y las políticas. Cabe señalar que cuando el profesional es reconocido e integrado su autopercepción es positiva y con ello su autoeficacia se ve incrementada, así también su compromiso. Por el contrario, el no-reconocimiento suele impactar en la seguridad personal y en toda posibilidad de posicionamiento activo-creativo.

En este último caso, se suelen activar estrategias de defensa y rechazo de las asignaciones o demandas. Como advierten Vescio et al. (2008), una CPA efectiva se despliega sobre el desarrollo de una identidad colectiva fuerte, a través del compromiso profesional de sus miembros y una autonomía profesional desarrollada bajo la regulación colegiada. Por su parte, Ávalos (2011) entrega evidenciadas que demuestran que el fortalecimiento del concepto de trabajo colaborativo es clave para mejorar la enseñanza y que el fortalecimiento de la identidad del grupo impacta positivamente en la elaboración de normas de interacción y en la responsabilidad comunitaria por el cumplimiento de esas normas.

\section{Evidencias de los impactos y efectividad de una CPA}

No cabe duda que las aristas que suscitan mayor interés en torno a las CPA son aquellas que tienen por finalidad identificar sus impactos en la gestión-organización de la escuela y en las prácticas de los profesores. Al respecto, y según Vanblaere y Devos (2016), Ávalos (2011), Vescio et al. (2008) y Hopkins (2008), la participación en CPA está efectivamente vinculada a la mejora de las prácticas de aula, a un mayor sentido de eficacia, motivación, compromiso y satisfacción. Estos autores, atribuyen estos impactos a la posibilidad de identificar dilemas-desafíos del trabajo, de interrogar las creencias y de priorizar-aprovechar las instancias de aprendizaje y de desarrollo profesional.

Otras investigaciones han abordado aspectos específicos de influencia, por ejemplo; sobre el rol crucial del Director para el desarrollo del trabajo y de la cultura colaborativa en la escuela, el incremento del compromiso y las condiciones de inserción que se generan para una inserción satisfactoria de los profesores principiantes (Vanblaere \& Devos, 2016). 
Sin embargo, en un contexto educativo donde prevalecen mecanismos de mercado, la competencia por recursos y un enfoque de rendición de cuentas, los impactos de una CPA en los resultados de los alumnos también han recibido particular atención de los diseñadores de política educativa. Como plantea Hopkins (2008) la relación alude a la generación de condiciones y procesos sustentables de gestión para la mejora, muchos de ellos asociados al profesor y sus prácticas.

Los estudios comparativos de Bolam (2005), Vescio et al. (2008) y los meta análisis de Ávalos (2011) para América Latina y Estados Unidos evidencian que aquellas escuelas donde se han desarrollado CPA los resultados de los estudiantes en evaluaciones nacionales relativas a distintas áreas del curriculum se incrementan. De hecho Bolam (2005) llega a igual conclusión y argumenta que cuando los profesores de primaria y secundaria informan de un mayor grado de participación y compromiso en la CPA de su escuela el nivel de progreso de los alumnos es mayor.

Pero no toda reunión de profesores constituye una comunidad profesional y a pesar de intentos por determinar medidas objetivas comparables ha sido difícil llegar a un acuerdo sobre una base o estándar que permita efectivamente establecer su logro. Lo que si queda en evidencia es que una CPA debe ser visualizada desde una perspectiva multidimensional en la que se incluyen capacidades de organización, personales e interpersonales. En la confección de la tabla No2 hemos recogido los aportes de los diversos autores hasta aquí referenciados a fin de determinar preliminarmente aspectos configurativos de una CPA.

Tabla 2: Perspectiva multid1imensional de una CPA: Ejes constitutivos

\begin{tabular}{|l|l|}
\hline \multicolumn{1}{|c|}{ Eje } & \multicolumn{1}{|c|}{ Contenidos } \\
\hline 1. Valores y visión compartida & $\begin{array}{l}\text { Cualidades, orientaciones y lecturas compartidas. Revisión y diálogo continúo sobre } \\
\text { los valores profesionales en pos de la construcción de capacidades. }\end{array}$ \\
\hline 2. Liderazgo distribuido & $\begin{array}{l}\text { Oportunidades para que diferentes profesionales desarrollen y puedan expresar } \\
\text { su capacidad de liderazgo en distintas áreas. }\end{array}$ \\
\hline 3. Confianza Organizacional & $\begin{array}{l}\text { Confianza, respeto y apoyo mutuo. Contribuir a la voluntad de para asumir riesgos } \\
\text { y adoptar innovaciones. Libertad para plantear y discutir posiciones, sentimientos y } \\
\text { frustraciones. }\end{array}$ \\
\hline 5. Compartir práctica profesional & $\begin{array}{l}\text { Claridad sobre aquello que la comunidad necesita aprender y de cómo debe } \\
\text { aprenderlo. Estrategias claras y acordadas para desarrollar aprendizaje. }\end{array}$ \\
\hline 6. Apertura, redes y alianzas & $\begin{array}{l}\text { La responsabilidad colectiva frente los aprendizajes de todos los estudiantes. } \\
\text { Desprivatizar el ejercicio o práctica. El trabajo deja de ser algo esencialmente privado para } \\
\text { convertirse en una cuestión de interés público. Estrategias y dispositivos de observaciones, } \\
\text { registros y devoluciones constantes entre docentes, que promueven e invitan a } \\
\text { reflexionar colectivamente sobre su práctica. }\end{array}$ \\
\hline
\end{tabular}


Aspecto interesante de la síntesis es que se concibe como un flujo o dinámica permanente de trabajo, diálogo y compromiso. Los distintos ejes o dimensiones asumen una naturaleza flexible en tanto pueden ser concretados conjuntamente. Los ejes además pueden servir como criterios de evaluación permanente de las acciones. Sobre este punto, cabe señalar que es la comunidad la encargada de elaborar la instancia, propuesta o proyecto a partir del cual se espera generar los procesos, aunque no necesariamente deben ser distintos a aquellos de los cuales ya se disponen. Por último, es la comunidad la que deberá también considerar la pertinencia de ayuda externa.

\section{Conclusiones}

Analizadas las dinámicas y complejidades del mundo rural, como así también las limitaciones y contradicciones de los enfoques predominantes a partir de los cuales se diseñan políticas para la educación hemos querido concluir en torno a las finalidades hacia las que debieran orientar los profesores rurales el desarrollo de una CPA. Para ello nos sustentamos en dos argumentaciones. En primer lugar, en aquellas que refieren a las evidencias de impactos y beneficios de las CPA en diversas dimensiones de gestión de una escuela y, en segundo, al marco epistémico sobre lo que es o debiera constituir como principio y propuesta de construcción de conocimiento.

En nuestra opinión, el primer argumento remite a las posibilidades de cambio que han sido constatadas tanto a nivel colectivo como individual, como también en una dimensión intersubjetiva como objetiva. El segundo, nos remite al plano de las definiciones, de las adhesiones y compromisos que la comunidad desea asumir o proyectar.

En razón de las consecuencias educacionales y profesionales que han generado más de 35 de años de políticas sustentadas bajo principios neoliberales, y particularmente aquellas orientadas al control y prescripción de los actores, sin que ello por cierto se haya traducido en mejores resultados, estimamos que la CPA deben tomar distancia de orientaciones o racionalidades técnicas que buscan reducirlas a modelos de actuación orientados a la obtención de resultados. En vista de las dinámicas y complejidades del mundo rural y las consecuencias extremas que en términos de exclusión ha tenido la globalización y el modelo económico, creemos que una CPA debiera asumirse como un proyecto social de cambio, una construcción o praxis pedagógico -educativa con profundas connotaciones identitarias, políticas y culturales orientada a generar las condiciones (saberes, compromisos y materiales) que se estiman necesarias para promover los cambios deseados.

Visto así, una CPA es efectiva cuando apunta al desarrollo profesional del profesor rural concebido como un proceso permanentemente articulado al devenir de la escuela, a la comunidad, a la cultura e historia local, aunque más específicamente cuando se generan paulatinamente procesos inteligentes y convergentes de aprendizaje organizacional. 
Bajo tales adhesiones es que podemos proyectar las finalidades particulares que debiera atender una CPA en la escuela rural. Pare ello será ineludible preguntarse por parte de sus integrantes qué involucra o qué significa educar en el mundo rural.

Estimamos en base a los análisis realizados que la propuesta educativa a desarrollar requerirá asumir inevitablemente los rasgos de una práctica para la libertad (Freire, 2007) en tanto invita a desafiar las acciones profesionales, particularmente a develar las contradicciones del modelo y, por sobre todo, dar a conocer a los sectores campesinos su situación de exclusión social. También coincidimos con el análisis realizado por Thomas y Hernández (2004) al señalar que debe orientarse al conocimiento, defensa y el rescate de las culturas locales así como el desarrollo de formas de cooperación y reciprocidad.

De esta forma y en coherencia con esta lectura, las finalidades generales de una CPA en este contexto debieran orientarse a:

Proyectar críticamente los márgenes de acción disponibles con la intención de evaluar la suficiencia o el aporte efectivo que realizan numerosos programas o agencias que intervienen actualmente la escuela rural. La CPA también debe dirigirse a preparar a los docentes para asumir y desarrollar una identidad como profesionales que les permitan realizar entre otras, lecturas críticas de los materiales curriculares y didácticos. En este último caso, será responsabilidad de los integrantes de la CPA prepararse y asesorarse para generar los conocimientos pertinentes y conducir las investigaciones que permitirán establecer, evaluar y priorizar necesidades y cursos de acción.

\section{Referências}

Avalos, B. Analizando las políticas a la luz del trabajo colaborativo y el desarrollo profesional. Docencia N. 60. 15-24. 2016.

Ávalos, B. Teacher professional development in Teaching and Teacher Education over ten years. Teaching and Teacher Education, 27, 10 - 20. 2011.

Bolam, R, McMahon, A., Stoll, L., Thomas, S., \& Wallace, M. Creating and sustaining professional learning communities. Research Report. Number 637. London, England: General Teaching Council for England, Department for Education and Skills. 2005.

Cornejo,J. Cómo acompañar a los centros Educativos en sus proyectos de cambio. Revista Foro Educacional. UCSH. N.11 pp. 9-67.2007.

Correa, E; Cividini, M. \& Fuentealba, R. Formación e inserción profesional: desafíos y pistas de facilitación para la profesionalización docente. Québec, Québec: PUQ. 2013.

Day, C. Pasión por enseñar. La identidad personal y profesional del docente y sus valores. Narcea: Madrid. 2011.

Egert, M. Aproximación a la Identidad Profesional de Docentes Rurales de la Provincia de Valdivia a través de Relatos de Vida. Tesis para optar al Título de Antropóloga y Licenciado en Antropología. Universidad Austral de Chile. 2007.

Ferrada, D. El ejercicio docente en contextos de alta vulnerabilidad y diversidad territorial, cultural y lingǘstica. Docencia N. 56, pp. 36-46. 2015.

Ferrada, D. Enlazando Mundos. Un modelo pedagógico que construye esperanzas de igualdad e inclusión en escuelas públicas. Rexe. N. 14,pp 37-52. 2008. 
Freire, P. La educación como práctica de la libertad. Siglo XXI. Montevideo: Uruguay. 2007.

Galaz, A. Evaluación e identidad profesional del profesor ¿Un juego de espejos rotos? Andamios. Volumen 12, numero 27, enero-abril, pp. 305-333. 2015.

Galaz, A. Fracturas de la identidad en la formación por competencias de los futuros profesores: análisis de una experiencia. Revista de Pedagogía. Vol. 35, Número. 97, 2014. pp. 52-70. 2014.

Galaz, A. El profesor y su identidad profesional ¿facilitadores u obstáculos del cambio educativo? Estudios Pedagógicos. Vol. 37, № 2, pp. 89-107. 2011a.

Galaz, A. Comunidades profesionales: una estrategia efectiva para la reflexión, el aprendizaje y la articulación entre profesores y formadores de profesores. En. Galaz, A; Fuentealba, R; Cornejo, J y Padilla, A. Estrategias reflexivas en la formación de profesores y de formadores de profesores. LOM Editores Santiago, pp. 83-114. 2011b.

Gobierno de Chile. Ley de Desarrollo Profesional N. 20903. Crea el sistema de desarrollo profesional docente y modifica otras normas. 2016.

Guerra, D. La Ruralidad Chilena Actual. Aproximaciones desde la Antropología (Comentarios). Chungará, Volumen 45, N. 3, 2013. 497-499. 2013.

Ho, D; Lee, M and Teng, Y. Exploring the relationship between school-level teacher qualifications and teachers' perceptions of school-based professional learning community practices. Teaching and Teacher Education. Vol. 54, 32-43.2016.

Hopkins, D. Hacia una buena escuela. Experiencias y lecciones. Fundación Chile. 2008.

Houssaye, J. Peut-on parler d'une problématisation pédagogique? Recherche et Formation, Institut National de Recherche Pédagogique. N. 48, 107-117. 2005.

Korthagen, F. (2010). La práctica, la teoría y la persona en la formación del profesorado. Revista Interuniversitaria de Formación del Profesorado. 68 (24,2), 83-101. 2010.

Krichesky, G. y Murillo, J. Las Comunidades Profesionales de Aprendizaje. Una Estrategia de Mejora para una Nueva Concepción de Escuela. REICE. Vol. 9, núm. 1, 2011, pp. 65-83

Leyton, T. Las políticas de educación rural en Chile: cambio y continuidad. Comunicación presentada en el Congreso de la Asociación Latinoamericana de Sociología 2013, Santiago de Chile. 2013.

Ministerio de Desarrollo Social. Resultados encuesta CASEN 2015. Disponible: <http://www. ministeriodesarrollosocial.gob.c1/resultados-encuesta-casen-2013/>. 2015.

Mineduc. Decreto 968. Exento autoriza reuniones en microcentro para profesores de escuelas rurales. 2012.

Mineduc. Estadísticas del sistema educativo. 2016.

Montecinos, C. Desarrollo profesional docente y aprendizaje colectivo. Psicoperspectivas. vol. II, pp. 105-128. 2003.

OCDE. TALIS. Informe global. 2009.

OCDE. TALIS. Informe Chile. 2013.

Programa de las Naciones Unidas para el Desarrollo. Informe de Desarrollo Humano Rural. Chile. PNUD. 2008.

OREALC-UNESCO. Antecedentes y criterios para la elaboración de políticas docentes en América Latina y el Caribe. Unesco: Santiago, 2013.

Peirano, C; Puni, S y Astorga, M. Educación rural: oportunidades para la innovación. Cuadernos de Investigación Educativa, Vol. 6, N. 1, 2015, Montevideo (Uruguay). 53-70. 2015. 
Comunidades profesionales de aprendizaje: oportunidades de desarrollo profesional en la exclusión y diversidad rural

Pino, F. Globalización, paisaje y vivienda rural. Revista de urbanismo N. 14, junio. Disponible: <file://C:/Users/Profesor/Desktop/Profesor\%20rural/Pino\%20Globalizaci\%C3\%B3n,\%20 paisaje\%20y\%20vivienda\%20rural.html>. 2004.

Senge, P. La quinta disciplina. Escuelas que aprenden. Norma Editores. 2004.

Thomas, C y Hernández, R. El rol del profesor en la educación rural. Presentación al V Congreso de Antropología. San Felipe. 2004.

Vanblaere, B. and Devos, G. Relating school leadership to perceived professional learning community characteristics: A multilevel analysis. Teaching and Teacher Education. 57. 26-38. 2016.

Vescio,V; Ross,D; Adams, A. A review of research on the impact of professional learning communities on teaching practice and student learning. Teaching and Teacher Education. 24 80-91.2008.

Williamson, G. Estudios sobre la educación para la población rural en Chile. En Educación para la población rural en Brasil, Chile, Colombia, Honduras, México, Paraguay y Perú. Proyecto FAO-UNESCODGCS/ ITALIA-CIDE-REDUC. Roma: FAO-UNESCO. 2004.

\section{Notas}

${ }^{1}$ Según datos que nos entregan los reportes oficiales al año 2016 se han constituido un total de 375 Microcentros (Mineduc, 2016).

* Acadêmico do Instituto de Ciências da Educação da Universidade Austral do Chile, Região dos Rios, Chile.

** Acadêmica do Instituto de Ciências da Educação da Universidade Austral do Chile, Região dos Rios, Chile.

*** Acadêmica da Faculdade de Ciências da Educação da Universidade Libre, Bogotá., Colômbia.

\section{Correspondência}

Alberto Galaz - Instituto de Ciencias de la Educación. Universidad Austral de Chile. Campus Isla Teja S/N. Región de los Rios, Chile.

E-mail: betogalazr@gmail.com - claudia.contreras@uach.cl-nadiap.acostam@unilibrebog.edu.co

Recebido em 04 de abril de 2017

Aprovado em 24 de outubro de 2017 
